The Astrophysical Journal, 234:L1-L7, 1979 November 15

(c) 1979. The American Astronomical Society. All rights reserved. Printed in U.S.A.

\title{
A HIGH-SENSITIVITY X-RAY SURVEY USING THE EINSTEIN OBSERVATORY AND THE DISCRETE SOURCE CONTRIBUTION TO THE EXTRAGALACTIC X-RAY BACKGROUND
}

\author{
R. Giacconi, J. Bechtold, G. Branduardi, W. Forman, J. P. Henry, C. Jones, E. Kellogg, \\ H. van der LaAn, ${ }^{1}$ W. Liller, ${ }^{2}$ H. Marshall, ${ }^{3}$ S. S. Murray, J. Pye, ${ }^{4}$ \\ E. Schreier, W. L. W. Sargent ${ }^{5}$ F. Seward, and H. Tananbaum \\ Harvard-Smithsonian Center for Astrophysics, Cambridge, Massachusetts \\ Received 1979 June 26; accepled 1979 August 8
}

\begin{abstract}
Surveys of two fields have been carried out utilizing the $\mathrm{X}$-ray imaging instruments of the Einstein Observatory to a limiting flux of $1.3 \times 10^{-14} \mathrm{ergs} \mathrm{cm}^{-2} \mathrm{~s}^{-1}$ in the $1-3 \mathrm{keV}$ band, a several hundred-fold increase in sensitivity with respect to previous surveys. Forty-three objects are observed with positional accuracies varying from $5^{\prime \prime}$ to $60^{\prime \prime}$. Optical and radio studies of candidate counterparts are described. The number-intensity relationship for extragalactic sources is evaluated, and the consequences of our findings are discussed with particular emphasis on the discrete source contribution to the extragalactic X-ray background.
\end{abstract}

We conclude that a large fraction of the background is due to previously unresolved discrete sources.

Subject headings: cosmic background radiation $-\mathrm{X}$-rays: sources

\section{INTRODUCTION}

The existence of an isotropic X-ray background was established in the rocket flight which discovered extrasolar X-ray sources (Giacconi et al. 1962). At energies above $2 \mathrm{keV}$, the high degree of isotropy and the lack of correlation with galactic features strongly suggest that the bulk of the background is of extragalactic origin; a significant fraction must come from $z>1$ (see Schwartz 1978). At energies below $1 \mathrm{keV}$ the background is not isotropic and there appears to be a substantial contribution from galactic emission (see reviews by Tanaka and Bleeker 1977; Bunner and Sanders 1979).

The origin of the background is attributed theoretically to either (1) diffuse emission or (2) the unresolved contribution of individual sources. Two examples of theories in the first category are the suggestions that the background may be due either to the Compton interaction of high-energy electrons with the microwave background radiation (Felten and Morrison 1966), or to thermal bremsstrahlung emission from a hot intergalactic gas (Silk 1973; Field and Perrenod 1977). Recent measurements of the spectrum of the background from the $H E A O \perp \mathrm{A}-2$ experiment have been interpreted as supporting the existence of such intergalactic gas (Boldt 1978).

\footnotetext{
${ }^{1}$ Leiden Observatory, The Netherlands.

${ }^{2}$ Visiting Astronomer, Cerro Tololo Inter-American Observatory, supported by the National Science Foundation under contract NSF-C868.

${ }^{3}$ Smithsonian Predoctoral Fellow.

${ }^{4}$ University of Leicester, England.

${ }^{5}$ Hale Observatory, California Institute of Technology, operated jointly by CIT and the Carnegie Institution of Washington.
}

On the other hand, the discovery of many classes of extragalactic $\mathrm{X}$-ray emitters, including emission-line galaxies, Seyfert galaxies, BL Lacertae objects, N galaxies, quasars, and clusters of galaxies, suggests the possibility that the summed contributions of more distant objects of these same classes may give rise to an as yet unresolved component. As early as 1973, Setti and Woltjer pointed out that if all QSOs emitted $\mathrm{X}$-rays at a constant $\mathrm{X}$-ray to optical luminosity ratio equal to that of $3 \mathrm{C} 273$ (the only X-ray emitting QSO then known), then the integrated emission from these objects could well account for most of the background.

New capabilities for investigating this problem were provided by the launch in 1978 November of an imaging X-ray telescope on the Einstein Observatory (for details, see Giacconi et al. 1979). Our observational program consists of obtaining deep X-ray exposures in selected fields; a sensitivity of $1.3 \times 10^{-14} \mathrm{ergs} \mathrm{cm}^{-2} \mathrm{~s}^{-1}$ in the $1-3 \mathrm{keV}$ range can be achieved for the detection of sources with angular extent $\leq 60^{\prime \prime}$. This sensitivity permits us to extend the observed number-intensity distribution by 3 decades in flux, and to study the nature and types of sources contributing to the background by examining their optical and radio counterparts. This approach differs from previous attempts to determine the nature of the $\mathrm{X}$-ray background through its spectrum or point-to-point fluctuations over an angular scale of degrees. The imaging capabilities and the thousand-fold increase in sensitivity of the Einstein Observatory make possible our more direct approach.

Since our main interest is to investigate the nature of the extragalactic X-ray background, we selected survey regions with low neutral-hydrogen column density at galactic latitude $>20^{\circ}$ with no known $\mathrm{X}$-ray source with intensity $>1.7 \times 10^{-11} \mathrm{ergs} \mathrm{cm}^{-2} \mathrm{~s}^{-1}$ in the $2-6 \mathrm{keV}$ 
range, or peculiar optical or radio source. We have $\therefore$ obtained data on two such fields; in what follows, we describe our observations and discuss our preliminary findings.

Several exposures were used in a mosaic fashion to cover a deep survey region with the imaging proportional counter (IPC) and the high-resolution image detector (HRI); the total exposure times ranged from 27,000 to $56,000 \mathrm{~s}$ for IPC observations and from 8000 $52,000 \mathrm{~s}$ for HRI observations. Owing to overlaps of fields of view and various mission-related constraints, the exposure is far from uniform over the survey region.

A computerized detection system was used to establish the existence of sources in each of the individual fields. The algorithm is based on the use of a sliding bin, of $2.4 \times 2.4$ for the IPC and of $12^{\prime \prime} \times 12^{\prime \prime}, 24^{\prime \prime} \times$ $24^{\prime \prime}$, and $36^{\prime \prime} \times 36^{\prime \prime}$ for the HRI, to measure the number of counts contained in a detection cell. The sliding step of the detection cell is one-third of the bin width. The computer program establishes a background level for each of the observations by measuring the actual number of counts in the field and establishes an appropriate threshold for detection based on the requirement that the number of spurious events predicted from random fluctuations anywhere in the field be less than 1 .

Spurious sources may be found with this technique (particularly at the lowest intensity) if discrete highbackground regions and/or detector nonuniformities are present. In order to ascertain the magnitude of this effect for the HRI, we summed the results from a large number of independent fields without applying aspect determination. Effects due to the internal characteristics of the detector are revealed by this summation. We found several regions (localized within a few arcsec) in which the background exceeded the average $1.9 \times 10^{-4}$ counts $\mathrm{s}^{-1}$ (in $12^{\prime \prime} \times 12^{\prime \prime}$ ) by a factor of 5-10. Photons whose detector coordinates corresponded to these "hot spots" were eliminated from further analysis. In the course of an observation, spacecraft drift is on the order of $0.5-1^{\prime}$. Thus this deletion reduces the sensitivity to each point in the sky by an insignificant amount. Large-scale detector background uniformity, on the other hand, was determined to be better than $10 \%$ over the entire useful field prior to launch. In-flight measurements of background uniformity have been made to show that a negligible increase in threshold is necessary to ensure that sources are not spuriously detected as a result of small nonuniformities.

For the IPC, it has not yet been possible (at this early stage in the mission) to determine all systematic background variations. Large-scale nonuniformity was taken into account by a first-order modeling of the background, which was assumed to depend only on the angular distance from the center of the detector. In order to evaluate residual small-scale effects, each of the fields in the survey was observed twice with the IPC, with a $\sim 5^{\prime}$ offset in the pointing position. Each set of observations was independently analyzed and the results cross-correlated. There do not appear to be any spurious detection of sources that satisfy the threshold criteria and are due to such systematic effects.

The above considerations pertain to the problem of establishing the existence of a weak source anywhere in the field on the basis of either IPC or HRI observations alone. Cross-correlation between IPC and HRI results allows us to confirm the existence of weak sources and to compare counting rates in the two detectors.

The HRI is equal to the IPC in sensitivity for softpoint sources and is less sensitive for extended and hard sources. However, the HRI detections, even at low signal-to-noise levels, yield accurate positions. From observations of X-ray sources with known optical counterparts, we estimate that the uncertainty in systematic corrections for the entire HRI/optical system is about $3^{\prime \prime}$. The error budget for weak sources is dominated by statistical uncertainty in the determination of the centroid of the photon distribution. The total positional errors (which are shown in Figs. 1-4) also include the uncertainty due to plate measurement errors; the resultant error radius for HRI positions is typically $\sim 5^{\prime \prime}$. The errors in the IPC positional determinations, on the other hand, are due mainly to the current imprecision in the transformation from electrical detector coordinates to sky coordinates. We estimate this error to be $60^{\prime \prime}$ radius.

In Tables 1 and 2 we summarize the information for the sources detected in the two deep surveys. The first column gives the source position as determined by the HRI detection (when available). We denote all Einstein sources by $1 \mathrm{E}$, followed by right ascension in hours, minutes, and seconds, and declination in degrees, minutes, and the truncated fraction of minutes. The second and third columns give the observed IPC counting rates in units of $10^{-3}$ counts $\mathrm{s}^{-1}(1-3 \mathrm{keV})$ after subtraction of the background and corrections for vignetting and exposure effects. In column four, the average rate from the previous two determinations is given. In column five, the observed HRI counting rate, in units of $10^{-3}$ counts $\mathrm{s}^{-1}(0.1-4 \mathrm{keV})$ after background subtraction, is given.

In the column labeled "Optical Candidate" the visual magnitude of candidate objects estimated from available optical data is given as described in the following section. Those objects whose nature is known from follow-on optical studies are further described. We also list radio fluxes as described below.

Figures 1, 2, 3, and 4 (Plates L1-L4) are the finding charts for each of the sources listed in Tables 1 and 2 . Error circles of appropriate diameters are drawn on the KPNO $4 \mathrm{~m}$ blue plate of the Draco region and the Palomar Observatory 48 inch $(1.2 \mathrm{~m})$ Schmidt blue plate of the Eridanus region. One source (1E $170757+$ 7054.9, Table 1), whose position is poorly known because of confusion problems in the IPC, has been omitted.

\section{OPTICAI AND RADIO DATA}

The optical photographic plates available to us included for Eridanus a CTIO $4 \mathrm{~m} U$ plate (lim. mag = 20.5), $B$ and $R$ Palomar Schmidt plates (lim. mag 22.0 
ind and 21.5), an $R$ Curtis Schmidt plate (lim. mag 21.0); $\therefore$ for Draco, $B$ and $R$ KPNO $4 \mathrm{~m}$ plates (lim. mag 22.0), $B$ and $R$ Palomar Schmidt plates (lim. mag 22.0 and 21.5), and one Agassiz $1.5 \mathrm{~m} U$ plate (lim. mag 17.5). On Objects that are within or close to the error circles 1 - (about $20 \%$ larger radius) given by the Einstein $\mathrm{Ob}$ servatory were examined for unusual properties-extreme blueness, ultraviolet excess, nonstellar appearance. From the photographic plates, we have derived approximate blue, red, and ultraviolet magnitudes for the most likely candidates. The primary source of magnitude calibrations was the Palomar Sky Survey prints for which star image diameters have been calibrated (Liller and Liller 1975; King and Raff 1977). $B$ magnitudes are accurate to $\pm 0.3 \mathrm{mag}$; calculated $B-$ $V$ values range in reliability from \pm 0.2 for the brightest stars to \pm 0.5 for the faintest.

Several radio sources coinciding with $\mathrm{X}$-ray sources

TABLE 1

\section{DRACO SURVEY}

\begin{tabular}{|c|c|c|c|c|c|}
\hline Name & IPC 29 & IPC 3670 . & $\mathrm{Av} \cdot \mathrm{IPC}$ & HRI & Optical Candidate \\
\hline 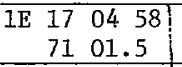 & & & $\begin{array}{l}(.53 \pm \\
.16)\end{array}$ & $\begin{array}{l}1.2 \pm \\
0.3\end{array}$ & $B=17.5, B-V=+0.3$, QSO $Z=2.0$ (Sargent), no proper motion. \\
\hline $\begin{array}{cccc}\text { IE } & 17 & 06 & 06 \\
71 & 07.2 \\
\end{array}$ & & & & $\begin{array}{l}1.11 \pm \\
.30\end{array}$ & $B=16.0, B-V=+0.7, G-t y p e$ star (Sargent), UX-Ari-type(?) \\
\hline 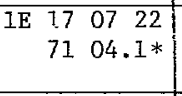 & $\begin{array}{l}.98 \pm \\
.26\end{array}$ & $\begin{array}{l}1.16 \pm \\
.29\end{array}$ & $\begin{array}{l}1.05 \pm \\
.20\end{array}$ & & $\begin{array}{l}\text { (a) } B=18.2, B-V=+0.9 \text {. Late type star (Sargent), no proper mo- } \\
\text { tion; (b) } B=19 \text {, neutral colored galaxy with reddish stellar } \\
\text { nucleus. Also, 4-6 faint stellar objects, B from } 21-22 \text {. }\end{array}$ \\
\hline $\begin{array}{llll}1 \mathrm{E} & 17 & 07 & 25 \\
& 70 & 44.5\end{array}$ & $\begin{array}{l}1.73 \pm \\
.39\end{array}$ & & & & $\begin{array}{l}\text { (a) } \mathrm{B}=16.0, \mathrm{~B}-\mathrm{V}=+1.0 \text {, stellar spectrum approx. } \mathrm{KO}(\mathrm{L} i 1 \mathrm{ler}) \text {, no } \\
\text { proper motion; (b) } \mathrm{B}=14.9, \mathrm{~B}-\mathrm{V}=1.0, \mathrm{~V}=7000 \mathrm{~km} \mathrm{~s}^{-1}(\mathrm{Liller}) \text {, com- } \\
\text { pact galaxy, no proper motion; (c) } \mathrm{B}=18.2, \mathrm{~B}-\mathrm{V}=+0.6 \text {, no proper } \\
\text { motion. }\end{array}$ \\
\hline IE $\begin{array}{rll}17 & 07 & 57 \\
70 & 54.9\end{array}$ & $\begin{array}{l}1.79+ \\
.40\end{array}$ & & & & Poor position, positional error $>60^{\prime \prime}$ \\
\hline $\begin{array}{llll}\mathrm{E} & 17 & 08 & 05 \\
& 71 & 18.4\end{array}$ & $\begin{array}{l}.85 \pm \\
.26\end{array}$ & & & & $\begin{array}{l}\text { (a) } \mathrm{Blue} S \mathrm{Sb} \text { galaxy, } \mathrm{B} \cong 17.5, \mathrm{Z}=0.0039, \lambda 3727[0 \text { II] in emission } \\
\text { (Sargent), no proper motion; }(\mathrm{b}) \mathrm{B}=18.4, \mathrm{~B}-\mathrm{V}=+0.6 \text {, metal poor } \\
\text { star (Sargent), no proper motion; }(\mathrm{c}) \mathrm{B}=19.8, \mathrm{~B}-\mathrm{V}=+0.6 \text {, no } \\
\text { proper motion; }(\text { d) } \mathrm{B} \approx 21, \mathrm{~B}-\mathrm{V}=+1.0 .\end{array}$ \\
\hline 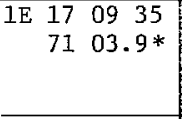 & $\begin{array}{l}.94 \pm \\
.25\end{array}$ & $\begin{array}{l}1.21 \pm \\
.29\end{array}$ & $\begin{array}{l}1.03 \pm \\
.19\end{array}$ & & $\begin{array}{l}\text { (a) } B=18.0, B=V=+0.4 \text { compact galaxy, small redshift } \lambda 3727[0 \text { II] } \\
\text { in emission (Sargent), no proper motion (b) } B=17.6, B-V \cong 0.5 \text {, no } \\
\text { proper motion (c) } B=18.7, B-V=+0.5 \text {, no proper motion; } \\
\text { (d) } B=19, B-V \simeq+0.8, \text { small diffuse object, no proper motion. }\end{array}$ \\
\hline $\begin{array}{llll}1 \mathrm{E} & 17 & 09 & 36 \\
& 71 & 15.2\end{array}$ & $\begin{array}{l}.87 \pm \\
.24\end{array}$ & & & & $\begin{array}{l}\text { (a) } B=19.1, B-V=+0.6 \text {, late type star (Sargent), no proper mo- } \\
\text { tion; (b) } B=18.1, B-V=-0.1 \text {, A type (Sargent), no proper motion; } \\
\text { (c) } B=16.7, B-V=+0.7 \text {, no proper motion; }(d) B=20.5 \text {, } B-V=+0.7 \\
\text { object. }\end{array}$ \\
\hline $\begin{array}{llll}1 \mathrm{E} & 17 & 09 & 57 \\
& 71 & 00.1 \\
\end{array}$ & $\begin{array}{l}1.90+ \\
.30\end{array}$ & $\begin{array}{l}1.51 \pm \\
.31\end{array}$ & $\begin{array}{l}1.71 \pm \\
.21\end{array}$ & $\begin{array}{l}.46 \pm \\
.12 \\
\end{array}$ & $\mathrm{~B}=12.0, \mathrm{~B}-\mathrm{V}=+0 . \overline{5}, \mathrm{G}-$ type star (Sargent) \\
\hline 1E $\begin{array}{lll}17 & 09 & 58 \\
71 & 27.5\end{array}$ & & $\begin{array}{l}1.46 \pm \\
.30\end{array}$ & & & $\begin{array}{l}\text { (a) } B=18.1, B-V=+0.5, \text { G-type subdwarf (Sargent), no proper mo- } \\
\text { tion; (b) } B=20.8, B-V=+0.8 ;(c) B=18.9, B-V=+0.8 \text {, no proper motion; } \\
\text { (d) } B \cong 21 \text {, neutral colored galaxy. }\end{array}$ \\
\hline $\begin{array}{rlll}1 \mathrm{E} & 17 & 10 & 51 \\
70 & 55.3^{*} \\
\end{array}$ & $\begin{array}{l}1.17 \pm \\
.27\end{array}$ & $\begin{array}{l}1.10 \pm \\
.28\end{array}$ & $\begin{array}{l}1.13 \pm \\
.20\end{array}$ & $.77 \pm$ & $\begin{array}{l}\text { Stellar object with } \mathrm{B} \cong 21.5, \mathrm{~B}-\mathrm{V} \cong+1.0 \text { (Schild, Liller). Additional } \\
\text { faint galaxies }(\sim 22-23 \mathrm{~m}) \text { (Kristian-Young-Westphal). }\end{array}$ \\
\hline $\begin{array}{llll}\mathrm{E} & 17 & 11 & 45 \\
& 71 & 16.0 \\
\end{array}$ & & & & $\begin{array}{l}.78+.23 \\
.70+.24 \\
\end{array}$ & $\mathrm{~B}=17.5, \mathrm{~B}-\mathrm{V}=+0.2$, QSO $\mathrm{Z}=1.6$ (Sargent), no proper motion. \\
\hline 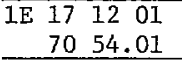 & & $\begin{array}{l}.99 \pm \\
.30 \\
\end{array}$ & & $\begin{array}{l}.73 \pm \\
.21 \\
\end{array}$ & $\mathrm{~B}=20.8, \mathrm{~B}-\mathrm{V}=+0.7$ \\
\hline $\begin{array}{llll}\mathrm{E} & 17 & 12 & 05 \\
& 71 & 11.9 \\
\end{array}$ & $\begin{array}{l}2.84 \pm \\
.33\end{array}$ & $\begin{array}{l}2.50 \pm \\
.33\end{array}$ & $\begin{array}{l}2.67 \pm \\
.23\end{array}$ & $\begin{array}{l}.99 \pm \\
.23 \\
\end{array}$ & $\begin{array}{l}\mathrm{B}=10.6, \mathrm{~B}-\mathrm{V}=+0.4 \text {, stellar spectrum; } \sim \mathrm{F} 2(\mathrm{Lill} \text { ) }) \text {, no proper } \\
\text { motion. }\end{array}$ \\
\hline 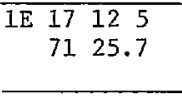 & & $.86 \pm$ & & & $\begin{array}{l}\text { (a) } \mathrm{B}=15.6, \mathrm{~B}-\mathrm{V}=+0.8 \text {, no proper motion. Six other objects } \\
\text { (brighter than } 17.1 \text {, }+0.8>\mathrm{B}-\mathrm{V}>+0.4 \text {, no proper motion). Two } \\
\text { objects, } \mathrm{B}=17.9 \text { and } \mathrm{B} \approx 20.1 \text {, both with } \mathrm{B}-\mathrm{V}=+0.2 \text {, no proper motion. }\end{array}$ \\
\hline 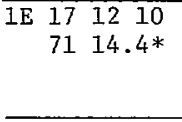 & $\begin{array}{l}2.63 \pm \\
.33\end{array}$ & $\begin{array}{l}2.82 \pm \\
.35\end{array}$ & $\begin{array}{l}2.69 \pm \\
.24\end{array}$ & & $\begin{array}{l}\text { (a) } B=18.0, B-V=+0.6, G \text {-type star (Sargent), no proper motion; } \\
\text { (b) } B=19.6, B-V=+0.9, M-t y p e \text { star (Sargent), no proper motion; } \\
\text { (c) } B \cong 20.5, B-V \cong+0.1 \text { compact galaxy (Sargent), no proper motion; } \\
\text { (d) radio source } 62.0 \pm 0.9 \mathrm{~mJ} \text { ( } 21 \mathrm{~cm}, 99 \pm 4 \mathrm{~mJ} \text { ( } 29 \mathrm{~cm} \text {, extended }\end{array}$ \\
\hline $\begin{array}{llll}E & 17 & 13 & 12 \\
& 71 & 11.1 \\
\end{array}$ & $\begin{array}{l}3.81 \pm \\
.39\end{array}$ & $.39 \pm$ & $\begin{array}{l}3.77 \pm \\
.28\end{array}$ & $\begin{array}{l}2.93+.35 \\
2.66+.58\end{array}$ & $\begin{array}{l}\mathrm{SAO} 8737 \\
\mathrm{~B}=9.7, \mathrm{~B}-\mathrm{V}=+0.8 \text {, Fo; proper motion } 0 ! 05 / \text { year }\end{array}$ \\
\hline IE $\begin{array}{lll}17 & 14 & 39 \\
71 & 26.8\end{array}$ & $\begin{array}{l}1.28 \pm \\
.37\end{array}$ & & & & 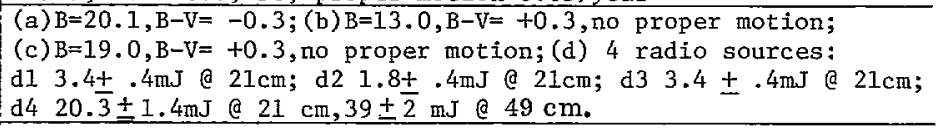 \\
\hline
\end{tabular}


IN have been detected in the two survey regions using the $\because$ Westerbork array for Draco and the Australian Naional Radio Astronomy Observatory for Eridanus. These results have provided precise positions which have made some of the X-ray identifications more $\rightarrow \rightarrow 1$ secure.

The spectrographic survey plates that we have used are a CTIO $4 \mathrm{~m}$ grating prism plate (lim. mag 21.5), an SRC Schmidt $U B$, thin-prism plate (lim. mag 21.0) (M. Smith, W. Forman, C. Jones, A. Fabian), a Curtis
Schmidt $B, 2^{\circ}$ prism (lim. mag 17.5), and an $R, 2^{\circ}$ prism (lim. mag 16.5) for Eridanus sources. No spectrographic survey plate was available for Draco. In addition, 23 of the brighter optical candidates were observed by members of the Optical and IR Division of the Harvard/Smithsonian Center for Astrophysics with the $Z$-machine attached to the Mount Hopkins $1.5 \mathrm{~m}$ reflector (M. Davis, A. Goldberg, J. Huchra, and W. Liller).

TABLE 2

ERIDANUS SURVEY

\begin{tabular}{|c|c|c|c|c|c|}
\hline NAME & IPC 3558 & IPC 3557 & Av. IPC & HRI & Optical Candidate \\
\hline $\begin{array}{rl}E 04 & 3655 \\
-16 & 37.5\end{array}$ & $\begin{array}{l}1.66 \pm \\
.44\end{array}$ & $\begin{array}{l}2.58 \pm \\
.63\end{array}$ & $\begin{array}{l}1.96 \pm \\
.36\end{array}$ & & $\begin{array}{l}\text { (a) } \mathrm{B}=19.2, \mathrm{~B}-\mathrm{V}=+0.1 \text {, blue featureless spectrum, extension to UV } \\
\text { (WD, } \mathrm{QSO} \text { ?); (b) } \mathrm{B}=19.8, \mathrm{~B}-\mathrm{V}=+0.8, \mathrm{p} 1 \mathrm{us} 6 \text { to } 8 \text { faint stellar ob - } \\
\text { jects at } \mathrm{B} \text { from } 21 \text { to } 22 \text {. }\end{array}$ \\
\hline 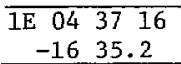 & & & & $\begin{array}{l}.87 \pm \\
.25\end{array}$ & $\mathrm{~B}=21.5, \mathrm{~B}-\mathrm{V} \leq+0.6, \mathrm{UV}$ bright stellar object. \\
\hline 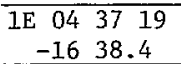 & & & & $.41 \pm$ & $\mathrm{B}=17.3, \mathrm{~B}-\mathrm{V}=+0.4$ featureless spectrum, no proper motion \\
\hline $\begin{array}{c}\mathrm{E} 043724 \\
-1629.9\end{array}$ & $\begin{array}{l}1.36 \pm \\
.37\end{array}$ & & & & $\begin{array}{l}\text { (a) } B=19.6, B-V=+0.1, \text { (b) } B=19.4, B-V=+0.7 \text {, no proper motion; } \\
\text { (c) } B=19.1, B-V=+1.3 \text {, no proper motion; (d) } B=21.2, B \simeq V=+0.5 \\
\text { diffuse object, UV bright. }\end{array}$ \\
\hline $\begin{array}{r}\mathrm{E} 043746 \\
-1620.3^{*}\end{array}$ & & $\begin{array}{l}2.63 \pm \\
.51\end{array}$ & & & $\begin{array}{l}\text { (a) } B=13.8, B-V=+0.5 \text {, Late } F \text { star (Liller), no proper motion; } \\
\text { (b) } B \text { s } 22, \text { (c) } B \approx 21 \text { neutra1 color galaxy; (d) } B=20.6, B-V=+0.4 ; \\
\text { (e) radio source } 5 \pm 3 \mathrm{~mJ}(6 \mathrm{~cm}\end{array}$ \\
\hline $\begin{array}{rl}\mathrm{E} 043753 \\
-16 & 12.3\end{array}$ & $\begin{array}{l}1.60 \pm \\
.43\end{array}$ & & & & $\begin{array}{l}\text { (a) } B=19.5, B-V=0.5 \text {, diffuse object; (b) } B=21.8, B-V=+0.8 \text {, stel- } \\
\text { lar object, featureless spectrun, } U V \text { bright. Two additional faint } \\
\text { objects, } B=22 \text {. }\end{array}$ \\
\hline $\begin{array}{rl}\text { IE } 043754 \\
-16 & 33.2 \\
\end{array}$ & & & $\begin{array}{l}(.84 \pm \\
.22)^{-}\end{array}$ & $\begin{array}{l}.93 \pm \\
.29\end{array}$ & $\mathrm{~B}=13.3, \mathrm{~B}-\mathrm{V}=+0.8, \mathrm{G} 5$ star, no proper motion \\
\hline 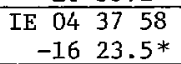 & $\begin{array}{l}2.10 \pm \\
.40\end{array}$ & $\begin{array}{l}1.73 \pm \\
.42\end{array}$ & $\begin{array}{l}1.92 \pm \\
.29\end{array}$ & & $\begin{array}{l}\text { (a) } \mathrm{B}=18.4, \mathrm{~B}-\mathrm{V}=+0.9 \text {, no proper motion; (b) } \mathrm{B}=20.4, \mathrm{~B}-\mathrm{V}=+0.8 \text {, } \\
\text { (c) } \mathrm{B}=22, \mathrm{~B} \sim \mathrm{V}<+0.5 \text {, UV brioht. }\end{array}$ \\
\hline $\begin{array}{rll}\text { IE } 04 & 38 & 00 \\
-16 & 32.3\end{array}$ & $\begin{array}{l}1.11 \pm \\
.34\end{array}$ & & & $.78 \pm$ & Empty field \\
\hline $\begin{array}{rl}\text { IE } 043801 \\
-16 & 28.3 \\
\end{array}$ & & $\begin{array}{l}1.67 \pm \\
.41\end{array}$ & & $\begin{array}{l}.55 \pm \\
.15 \\
\end{array}$ & Empty field \\
\hline 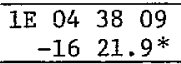 & $\begin{array}{l}2.29 \pm \\
.40\end{array}$ & $.93 \pm$ & $\begin{array}{l}1.54 \pm \\
.27\end{array}$ & $.82 \pm$ & $\mathrm{B}=21.5, \mathrm{~B}-\mathrm{V} \leq+0.6, \mathrm{~b}$ lue featureless spectrum \\
\hline $\begin{array}{rl}1 \mathrm{E} \quad 043811 \\
-16 & 38.2 \\
\end{array}$ & & $\begin{array}{l}(2.13 \pm \\
.58)\end{array}$ & & $\begin{array}{l}.32 \pm \\
.10\end{array}$ & $\begin{array}{l}\mathrm{B}=17.8, \mathrm{~B}-\mathrm{V}=-0.2 \text {, QSO } \mathrm{Z}=1.96 \text { (Liller, Smith), UV bright; no } \\
\text { proper motion }\end{array}$ \\
\hline 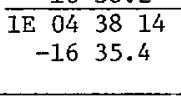 & & $\begin{array}{l}1.56 \pm \\
.40\end{array}$ & & & 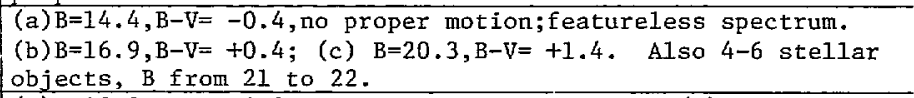 \\
\hline $\begin{array}{r}\text { IE } 043822 \\
-16 \quad 42.2^{*}\end{array}$ & $\begin{array}{l}1.94 \pm \\
.38\end{array}$ & $\begin{array}{l}1.25 \pm \\
.39\end{array}$ & $\begin{array}{l}1.60 \pm \\
.27\end{array}$ & & $\begin{array}{l}\text { (a) } B=19.2, B-V=+0.8 \text {, stellar, no proper motion; (b) } B=19.8 \text {, } \\
B-V=+0.9 \text { stellar, no proper motion; (c) } B \cong 19.5 \text {, diffuse blue } \\
\text { object; (d) } B=19.9, B-V=+1.0 \text {. }\end{array}$ \\
\hline 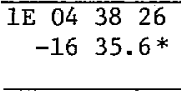 & $\begin{array}{l}4.07 \pm \\
.47\end{array}$ & $\begin{array}{l}4.42 \pm \\
.57\end{array}$ & $\begin{array}{l}4.21 \pm \\
.36\end{array}$ & $\begin{array}{l}1.16 \pm .15 \\
.83 \pm .14 \\
.50+.15 \\
\end{array}$ & $\begin{array}{l}\mathrm{B}=19.8, \mathrm{~B}-\mathrm{V}=+0.2, \mathrm{QSO} \mathrm{Z}=0.5(\mathrm{Smith}), \text { UV bright } \\
\text { Radio source } 10 \pm 2 \mathrm{~mJ} \text { a } 6 \mathrm{~cm}\end{array}$ \\
\hline $\begin{aligned} \mathrm{E} 043838 \\
-1641.0 \\
\end{aligned}$ & $\begin{array}{l}1.83+ \\
.37\end{array}$ & $\begin{array}{l}2.99 \pm \\
.51\end{array}$ & $\begin{array}{l}2.23 \pm \\
.30\end{array}$ & $\begin{array}{l}1.05 \pm .15 \\
.90+.17\end{array}$ & $\mathrm{~B}=13.1, \mathrm{~B}-\mathrm{V}=+0.7, \mathrm{G} 0$ Star, small proper motion \\
\hline $\begin{array}{r}1 E 043839 \\
-1634.8\end{array}$ & & & & $\begin{array}{l}1.03 \pm \\
.25\end{array}$ & $\begin{array}{l}B=17.1, B-V=+0.5, \text { stellar object, no proper motion. Featureless } \\
\text { spectrum, jet extending to } \mathrm{NE} \text { seen on both blue and red PS } \\
\text { plates; noton UV } 4-\mathrm{m} \text { plate }\end{array}$ \\
\hline $\begin{array}{rl}1 E & 043839 \\
-16 & 49.4 \\
\end{array}$ & & $\begin{array}{l}2.07 \pm \\
.46 \\
\end{array}$ & & $\begin{array}{l}.58 \pm \\
.21 \\
\end{array}$ & $\begin{array}{l}B=21.1, B-V=0.3, \text { stellar object with fainter, possibly } \\
\text { diffuse companion }\end{array}$ \\
\hline $\begin{array}{rr}0438 & 35 \\
-16 & 18.4 \\
\end{array}$ & & $.89 \pm$ & & $\begin{array}{l}.80+.21 \\
.64+.20 \\
\end{array}$ & $\begin{array}{l}\mathrm{B}=19.1, \mathrm{~B}-\mathrm{V}=+0.3 \text {, featureless spectrum; no proper motion, } \\
\mathrm{UV} \text { bright }\end{array}$ \\
\hline $\begin{array}{rll}04 & 38 & 49 \\
-16 & 21.9\end{array}$ & & $\begin{array}{l}1.28 \pm \\
.39\end{array}$ & & $\begin{array}{l}.50 \div .15 \\
.26+.10 \\
\end{array}$ & $\begin{array}{l}\mathrm{B}=21.0, \mathrm{~B}-\mathrm{V} \cong+0.4, \text { possibly diffuse object; radio source } \\
22+2 \mathrm{~mJ} \text { a } 6 \mathrm{~cm}\end{array}$ \\
\hline $\begin{array}{r}\text { IE } 043916 \\
-1622.5 \\
\end{array}$ & & & $\begin{array}{l}(.72+ \\
.22) \\
\end{array}$ & $\begin{array}{l}.67 \pm \\
.16\end{array}$ & $\mathrm{~B}=15.8, \mathrm{~B}-\mathrm{V}=+1.2 \mathrm{~K} 8-\mathrm{K} 9$ star; no proper motion \\
\hline $\begin{array}{rl}\mathrm{IE} 043947 \\
-16 & 20.5 \\
\end{array}$ & & & & $\begin{array}{l}.91 \pm \\
.26 \\
\end{array}$ & $\mathrm{~B}=13.7, \mathrm{~B}-\mathrm{V}=+0.7$, Late $\mathrm{F}$ (Liller), no proper motion \\
\hline $\begin{array}{r}\text { IE } 043953 \\
-1631.5 \\
\end{array}$ & & & & $.65 \pm$ & Empty field \\
\hline
\end{tabular}




\section{DISCUSSION}

In order to determine the contribution of the sources in Tables 1 and 2 to the extragalactic background, we convert the IPC count rates to flux $\left(\operatorname{ergs~cm}{ }^{-2} \mathrm{~s}^{-1}\right)$ in the $1-3 \mathrm{keV}$ range. The sources are weak and a determination of their individual spectra cannot be made using these data. However, by assuming a differential photon spectrum of the shape $A E^{-\alpha}$ with $\alpha=+1.4$ (the slope of the extragalactic background spectrum in the energy range of interest [Schwartz 1978]), without cutoff, the conversion factor is: 1 IPC count $\mathrm{s}^{-1}=$ $2.5 \times 10^{-11}$ ergs $\mathrm{cm}^{-2} \mathrm{~s}^{-1}(1-3 \mathrm{keV})$ with an estimated uncertainty of about $\pm 15 \%$ due to the combination of uncertainties in the incident spectrum (for $1 \leq \alpha \leq 2$, $0 \lesssim N_{\mathrm{H}} \lesssim 10^{21} \mathrm{~cm}^{-2}$ ) and in the detector gain and spectral resolution. In order to obtain a complete sample to some limiting minimum value, we have chosen sources with $1-3 \mathrm{keV}$ flux greater than $2.6 \times$ $10^{-14}$ ergs cm$~_{-2} \mathrm{~s}^{-1}$ in Draco and $3.9 \times 10^{-14} \mathrm{ergs} \mathrm{cm}^{-2}$ $\mathrm{s}^{-1}$ in Eridanus. Moreover, in order to reduce the effects of uncertainty in source strength, we have selected sources whose intensity is determined to better than 5 standard deviations (from the photon counting statistics), either in one detector field alone, or in the average of fluxes measured from overlapping IPC fields. This restriction reduces possible errors due to uncertainty in source intensity. It does, however, exclude some real sources, with fluxes greater than $2.6 \times 10^{-14} \mathrm{ergs}^{-2}$ $\mathrm{s}^{-1}$, but whose intensity is poorly known from the computation. We estimate that this procedure may lead to an underestimate of the source counts by as much as $30 \%$. Only sources found in a $32^{\prime} \times 32^{\prime}$ square surrounding the center of the field have been selected, in order to avoid large $(>15 \%)$ vignetting corrections or window support problems. We then removed from the sample four sources identified with stars on the basis of optical spectra. The remaining 10 sources in our sample are indicated by an asterisk in the first column of Tables 1 and 2, and constitute our selected sample. We believe that these are mainly extragalactic objects and that any contamination from unidentified stars is small. This is based on a number of considerations summarized below.

a) Spectral information, obtained by combining the data from our sample of 10 sources, indicates an average spectrum significantly harder than the spectrum of identified stellar sources. This indicates that these sources are not typical stars.

b) The ratio of $1-3 \mathrm{keV} \mathrm{X}$-ray emission to visible light emission for the possible optical counterparts listed in Tables 1 and 2 is too large for main-sequence stars of the same $B-V$ color. This is based on the results of Vaiana et al. (1979) in which the X-ray to optical luminosity versus $B-V$ color is given for stars found to be $\mathrm{X}$-ray sources.

c) Proper motion studies of all candidates in the X-ray error boxes brighter than $B=20 \mathrm{mag}$ were carried out to a sensitivity of $0.04 \mathrm{yr}^{-1}$, and no motions were found. From this we conclude that these objects are at least $100 \mathrm{pc}$ distant. The number of hot (thermally radiating) white dwarfs more distant than $100 \mathrm{pc}$ and still observable in the 1-3 keV energy band at our flux limit is less than 0.1 (Henry et al. 1976).

d) For some stars, particularly main-sequence spectral type $\mathrm{M}, \mathrm{X}$-ray emission is found to vary by at least a factor of 10 in flares of an hour's duration. When in such a flare state, these stars can have an $\mathrm{X}$-ray to visible light emission ratio consistent with the observed values for some source candidates. We have analyzed our data to search for flaring activity. Our sensitivity is such that we could detect changes of a factor of 3 in times of less than 3 hours. No evidence for such variability was found for any of the sample sources, indicating that flaring $\mathrm{M}$ stars are not likely to be contributing to our sample.

We calculate the contribution of discrete sources to the extragalactic isotropic background using the data from the 10 selected individual sources as described above. In calculating $N(>S)$, the number of sources per steradian with flux greater than $S$, we count the number of sources observed, corrected for sky coverage, at a given sensitivity. This is shown in Table 3 .

We obtain a value of $7.5(+2.9,-2.2) \times 10^{4}$ sources $\mathrm{sr}^{-1}$ with $S>2.60 \times 10^{-14}$ ergs $\mathrm{cm}^{-2} \mathrm{~s}^{-1}$. The uncertainty is the $68 \%$ confidence interval based on Poisson statistics in the number of sources observed. (See Murray and Pye 1979, in preparation for a more detailed discussion of the calculation of this uncertainty.) A systematic correction should be made to this value due to the effects of source confusion and Gaussian noise in the assigned source intensities (Murray 1977). The magnitude of these effects is estimated to be $\sim 20 \pm 10 \%$. This gives a best value of $6.3( \pm 2.6) \times$ $10^{4}$ sources $\mathrm{sr}^{-1}$ when we include both the systematic and statistical errors.

Assuming that these sources are part of a distribution which follows the number-flux relation given by $N(>S)=\gamma_{0}\left(S / S_{0}\right)^{-3 / 2}$, we can calculate the integral contribution of all sources (to our limiting flux level of $2.6 \times 10^{-14}$ ergs $\mathrm{cm}^{-2} \mathrm{~s}^{-1}$ ) to the background. The value for $V_{0}$ we calculate is $7.1 \pm 2.9$ (the number of sources per sr at a flux $S_{0}$ of $1.12 \times 10^{-11}$ ergs $\mathrm{cm}^{-2} \mathrm{~s}^{-1}=$ $1 \mathrm{UFU}(1-3 \mathrm{keV}))$. We can compare the normalization of the source distributions obtained from the Uhuru or Ariel 5 sky surveys (Murray 1977; Warwick and Pye 1978) with our result. The best values from these surveys extrapolated to $1-3 \mathrm{keV}$, assuming a background spectrum with slope -1.4 , are $11.0 \pm 2.6$ (Uhuru) and $12.6 \pm 2.5$ (Ariel 5) sources $\mathrm{sr}^{-1}$ at 1 UFU (Schwartz 1978), which clearly is in close agreement with our value even though the sources used to deter-

TABLE 3

Number of Sources Observed, Corrected for Sky Coverage, at a Given Sensitivity

\begin{tabular}{|c|c|c|c|}
\hline$\Delta S$ & $\begin{array}{l}\text { No. } \\
\text { Obs. }\end{array}$ & Coverage & $\begin{array}{c}\text { Corrected } \\
\text { No. }\end{array}$ \\
\hline 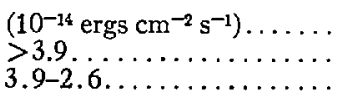 & $\ddot{7}$ & $\begin{array}{c}\left(1.73 \times 10^{-4} \mathrm{sr}\right) \\
1 \\
0.5\end{array}$ & $\begin{array}{l}77 \\
6\end{array}$ \\
\hline
\end{tabular}


20.3 mag could account for essentially all of the backmine $N_{0}$ are about 500 times weaker in flux. This $\therefore$ agreement indicates that the form for $N(>S)$ is valid ' over a large dynamic range in flux. This can be explained either in terms of a uniform distribution of relatively nearby objects in space, or in terms of -I cosmological density evolution making up for cosmological distance-volume effects. The first case occurs if we are observing objects within a small range of redshifts and a large range in luminosities, which seems unlikely in view of the optical work and following the considerations of Schwartz (1978). The second occurs if we are observing objects out to large redshifts $(z>$ 1) and the cosmological decrease in source counts is compensated for by evolutionary effects. For example, the number-flux relationship for active galaxies and QSOs (assuming a strong density evolution of the QSOs) can approximate a power law of slope $\sim-1.5$ over our observed flux interval.

The total extragalactic $\mathrm{X}$-ray background in the $1-3 \mathrm{keV}$ band is computed by integration of the spectrum given by Schwartz (1978), $N(E)=7.7 E^{-1.4}$ photons $\mathrm{cm}^{-2} \mathrm{~s}^{-1} \mathrm{sr}^{-1} \mathrm{keV}^{-1}$, over the $1-3 \mathrm{keV}$ interval. This gives $B_{t}=1.9 \times 10^{-8} \mathrm{ergs} \mathrm{cm} \mathrm{cm}^{-2} \mathrm{~s}^{-1} \mathrm{sr}^{-1}$. The observed number-flux relation given above shows that the background due to sources with $S>2.6 \times 10^{-14} \mathrm{ergs} \mathrm{cm}^{-2}$ $\mathrm{s}^{-1}$ is $4.9( \pm 2.1) \times 10^{-9} \mathrm{ergs} \mathrm{cm}^{-2} \mathrm{~s}^{-1} \mathrm{sr}^{-1}$, which corresponds to $26 \pm 11 \%$ of $B_{t}$.

We now consider the extrapolation of our observations to lower fluxes. We have observed sources fainter than $2.6 \times 10^{-14} \mathrm{ergs} \mathrm{cm}^{-2} \mathrm{~s}^{-1}$ which were not included in the $N(>S)$ determination because of their large intensity uncertainty as discussed previously. However, the existence of these sources is clearly established. Assuming that our derived number-flux relation holds to the smallest observed $S$, we can calculate the contribution of these fainter sources to the X-ray background. Taking $S_{m}=1.3 \times 10^{-14}$ ergs $\mathrm{cm}^{-2} \mathrm{~s}^{-1}$ (the faintest source observed with the IPC), we obtain $37 \pm$ $16 \%$ of the observed background due to discrete sources corresponding to flux levels already observed.

It is clear that if the discrete sources in our sample are active galaxies and quasars, we should extrapolate their contribution to even fainter fluxes corresponding to the faintest objects of this type whose existence is established on the basis of optical data. In order to do this, however, one must know the distribution of $L_{x} / L_{\text {opt }}$ for objects at a given magnitude, which we cannot derive from our data since we may be biased toward large $L_{x} / L_{\text {opt. }}$ Tananbaum et al. (1979), whose QSO sample is subject to a smaller and correctable bias in this respect, although possibly subject to severe selection effects, have shown that integration of the $\mathrm{X}$-ray fluxes expected from quasars brighter than $B \approx$ ground. Our observation that $37 \%$ of the $1-3 \mathrm{keV}$ background is due to discrete sources is consistent with the conclusion of Tananbaum et al. (1979), and we would expect that a significant fraction of the sources we have observed will be associated with quasars. In fact, we note that the source $1 \mathrm{E} 043826-1635.6$ has been found to be a QSO with redshift $z=0.5$. Further identifications will require improvements in the $\mathrm{X}$-ray source locations and optical spectra of possible candidates.

We have planned to carry out additional deep surveys, including one with a limiting sensitivity about 3 times fainter than that already achieved. With these observations we should be able to extend the numberflux distribution to the region where significant deviations from a simple power-law relation may occur. This is expected if quasars start to dominate the discrete source population since optical number counts for QSOs have a steeper slope than that which is currently observed for X-ray sources (Braccesi et al. 1979).

At this time, we feel justified in concluding that a substantial fraction of the extragalactic background is due to discrete sources. While previous discussions on this subject yielded estimates comparable to our quoted value, they were based on an extrapolation over at least three orders of magnitude in flux. Our results thus represent the first direct measurements of this quantity at fluxes low enough to yield a substantial contribution.

We gratefully acknowledge helpful discussions with Dr. G. Vaiana regarding X-ray emission from stars, and with Drs. D. A. Schwartz, Y. Avni, and G. Zamorani regarding different aspects of this Letter. We also wish to thank C. Kowal for obtaining several optical plates at the 48 inch Schmidt telescope at Mount Palomar; Schwarz and Bulger for a $U$ plate at the $1.5 \mathrm{~m}$ telescope at Agassiz Station; J. R. Katgert-Merkelijn, P. Katgert, and J. G. Robertson for radio data from Westerbork; A. E. Wright for radio data from Parkes; D. L. Jauncey, A. E. Wright, M. G. Smith, and B. A. Peterson for optical plates and spectroscopic results from the AngloAustralian Telescope; H. Gursky and R. Schild for CCD observations at Mount Hopkins; and J. Kristian, J. Westphal, and P. J. Young for CCD observations at the $5 \mathrm{~m}$ telescope at Mount Palomar. This research was sponsored under NASA contract NAS 8-30751. One of us (W. L. W. S.) received financial support from NSF Contract ASP-78-23795. John P. Pye acknowledges financial support of the U. K. Science Research Council.

The authors wish to thank the referee, Dr. John A. Bahcall, for the many critical and probing comments and suggestions which have aided us greatly in the preparation of this manuscript.

\section{REFERENCES}

Boldt, E. A. 1978, NASA Tech. Memo, No. 78106.

Braccesi, A., Zitelli, V., Bonoli, F., and Formiggini, L. 1979, Astr. $A$ p., submitted.

Bunner, A. N., and Sanders, W. T. 1979, A p. J. (Letters), 228, L19.

Felten, J. E., and Morrison, P. 1966, $A$ p. $J ., 146,686$

Field, G., and Perrenod, S. C. 1977, A p. J., 215, 717.

Giacconi, R., Gursky, H., Paolini, F. R., and Rossi, B. B. 1962, Phys. Rev. Letters, 9, 439.

Giacconi, R., et al. 1979, Ap. J., 230, 540.

Henry, J. P., Bowyer, S., Rapley, C. G., and Culhane, J. L. 1976, Ap.J. (Letters), 209, L29.

King, I. R., and Raff, M. I. 1977, Pub. A.S.P., 89, 120. 
Niller, M. H., and Liller, W. 1975, Ap. J. (Letters), 199, 133.

i Murray, S. S. 1977, Center for Astrophysics Preprint, No. 680.

in' Murray, S. S., and Pye, J. P. 1979, in preparation.

1O Schwartz, D. A. 1978, Proc. IAU/COSPAR Symposium on X-ray

Astronomy, Innsbruck, Austria, in press.

IOI Setti, G. and Woltjer, L. 1973, in IAU Symposium No. 55

-II $X$ - and Gamma-ray Astronomy, ed. H. Bradt and R. Giacconi (Dordrecht: Reidel), p. 208.

J. Bechtold, G. Branduardi, W. Forman, R. Giaccont, J. P. Henry, C. Jones, E. Kellogg, H. van der Laan, W. Liller, H. Marshall, S. S. Murray, J. Pye, W. L. W. Sargent, E. Schreier, F. Seward, and H. Tananbaum: Harvard/Smithsonian Center for Astrophysics, 60 Garden Street, Cambridge, MA 02138 

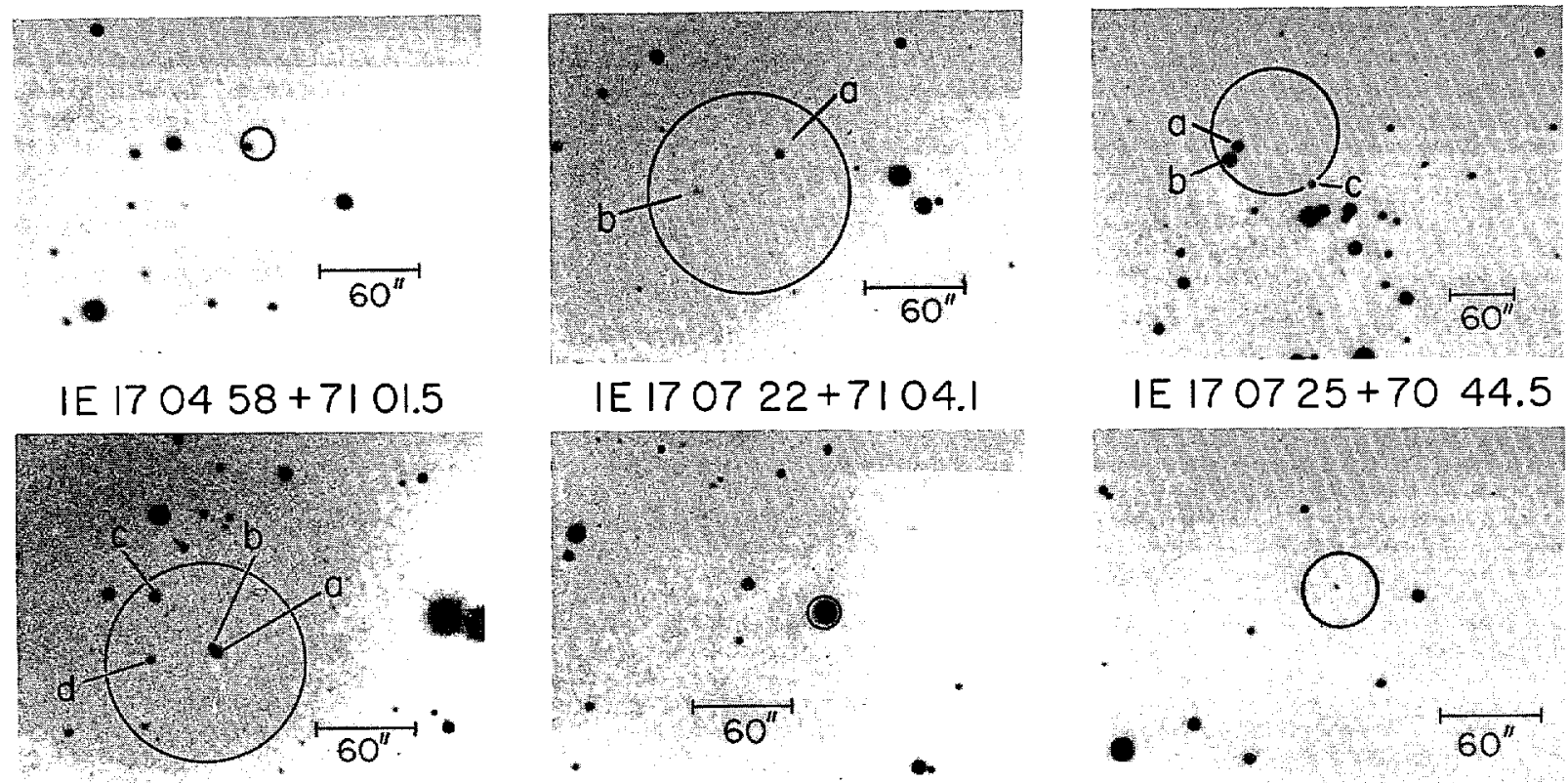

IE $170722+7104.1$

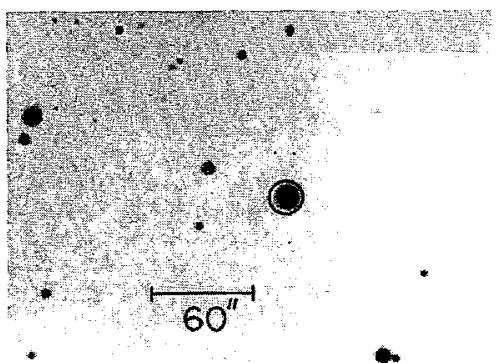

IE $170725+7044.5$

IE $170935+7103.9$
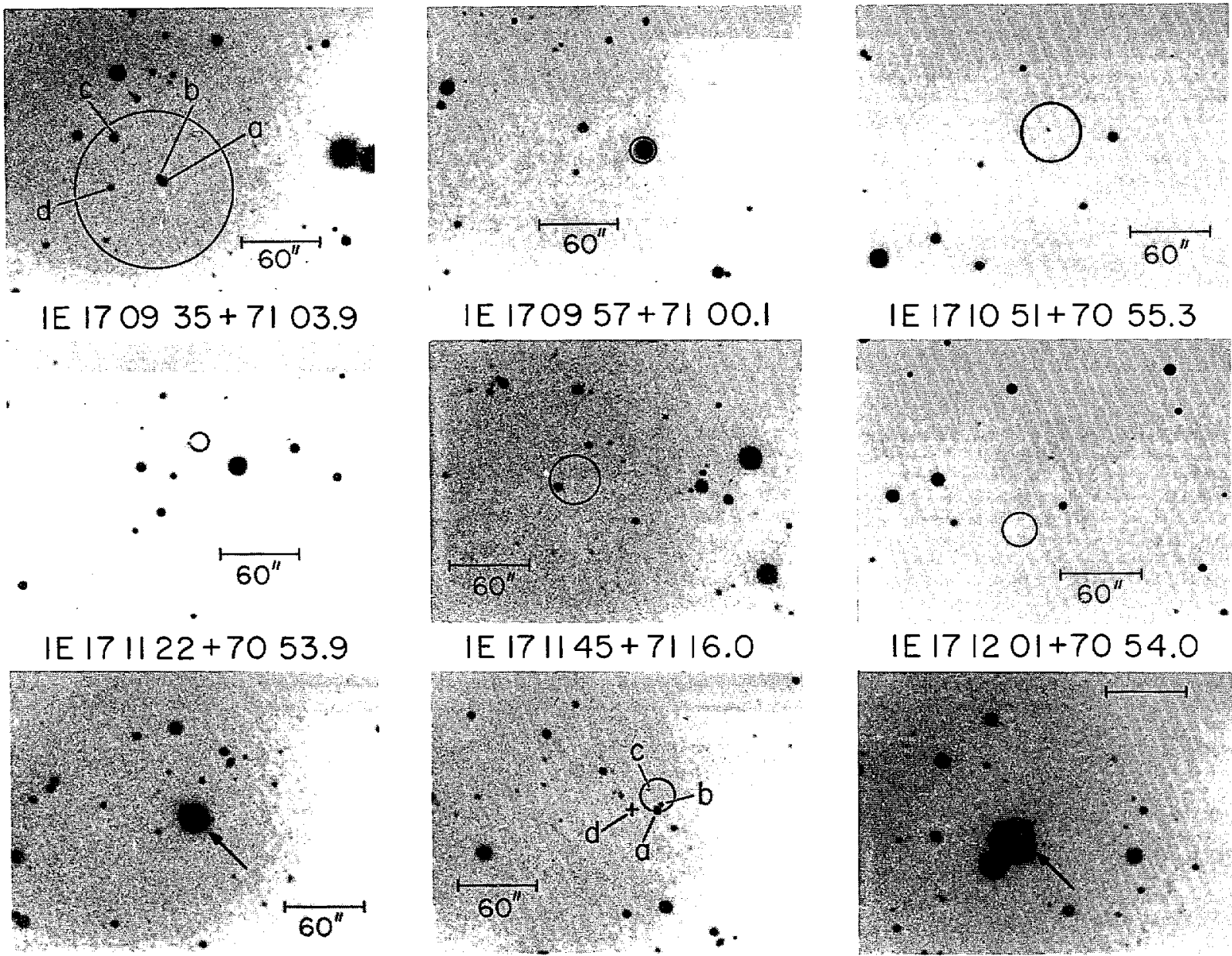

IE $171051+7055.3$

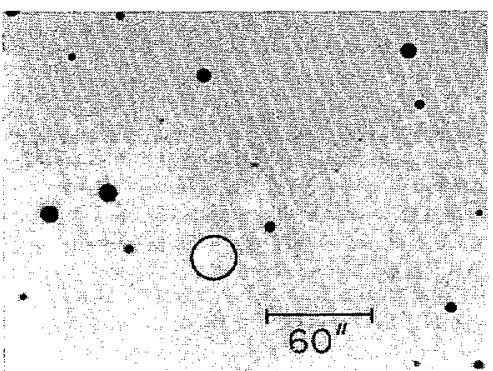

$|E 17| 145+7116.0$

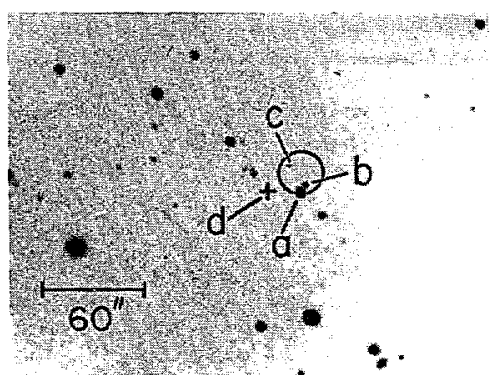

IE $171201+7054.0$

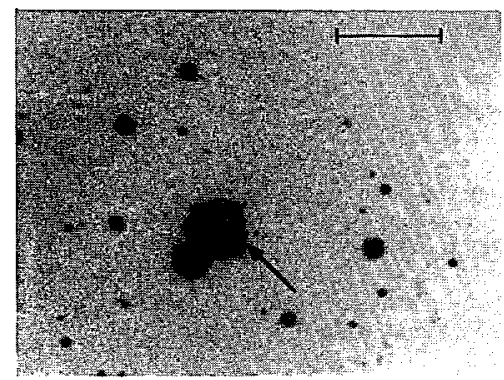

IE $171210+7114.4$

$|E 171312+71| 1.1$

$\mid E 171205+7111.9$

FIG. 1.-Finding charts for sources in the constellation Draco, as listed in Table 1

GiAcconi et al. (see page L2) 

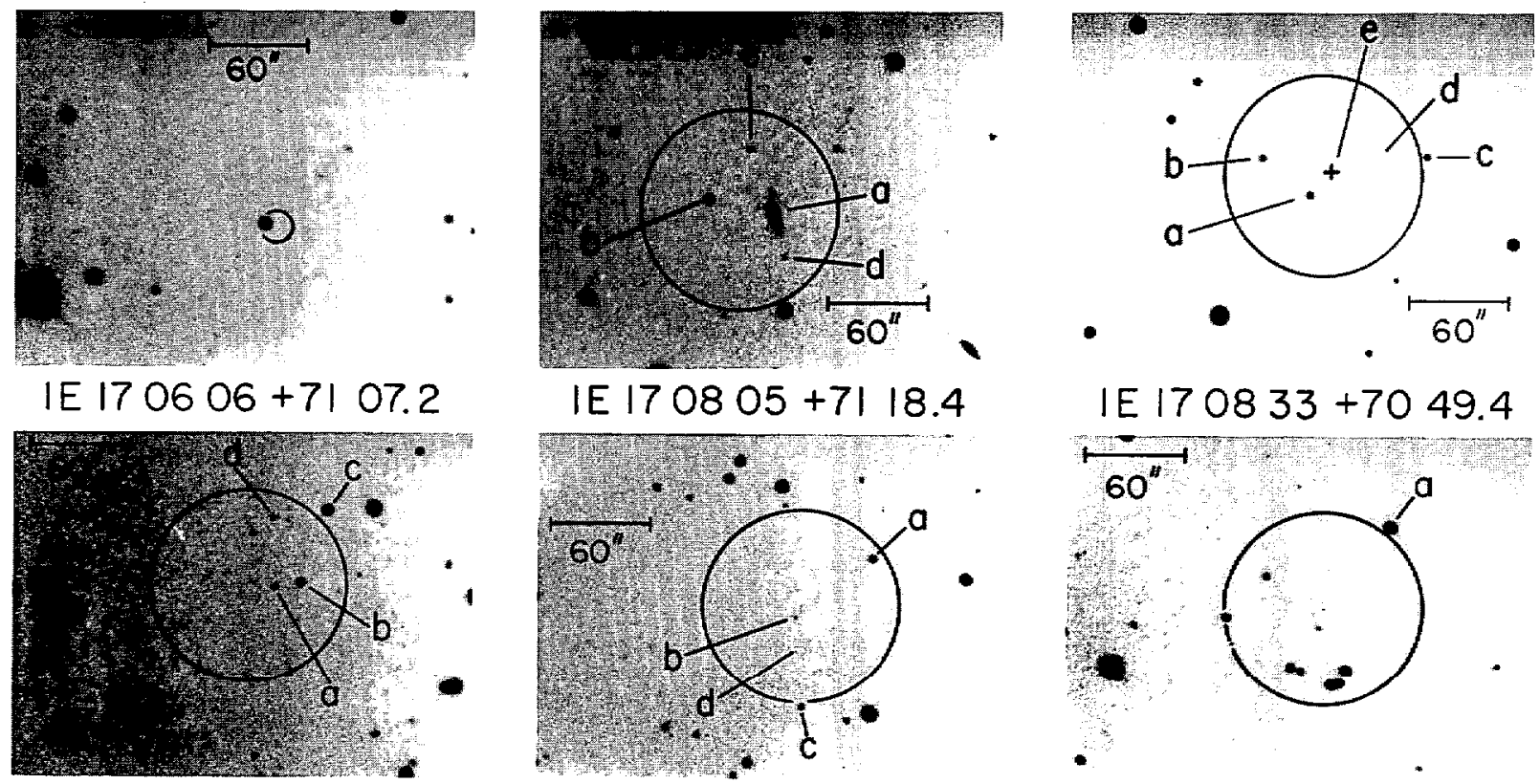

IE $170833+7049.4$

$|E| 70936+7 \mid 15.2$

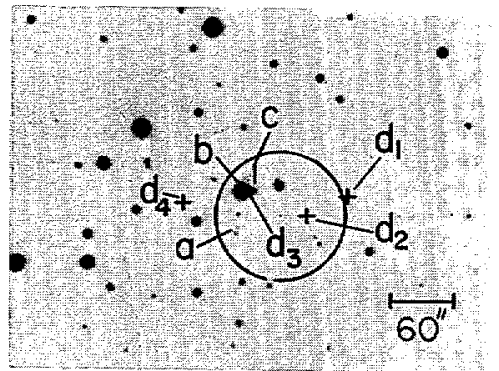

IE $170958+7 \mid 27.5$

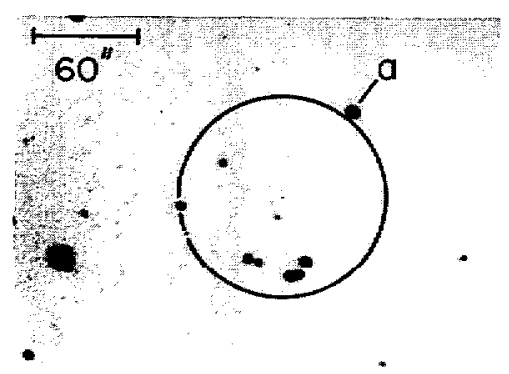

IE $171205+7125.7$

IE $171439+7126.8$

Fig. 2.-Continuation of finding charts for sources in the constellation Draco, as listed in Table 1

GIAcCONI et al. (see page L2) 
PLATE L3
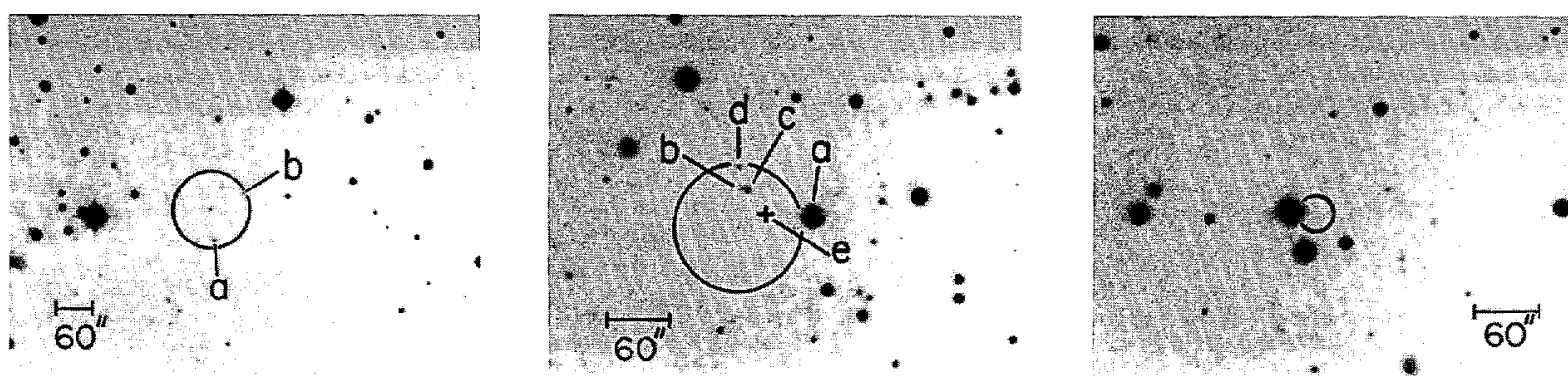

IE $043655-1637.5$
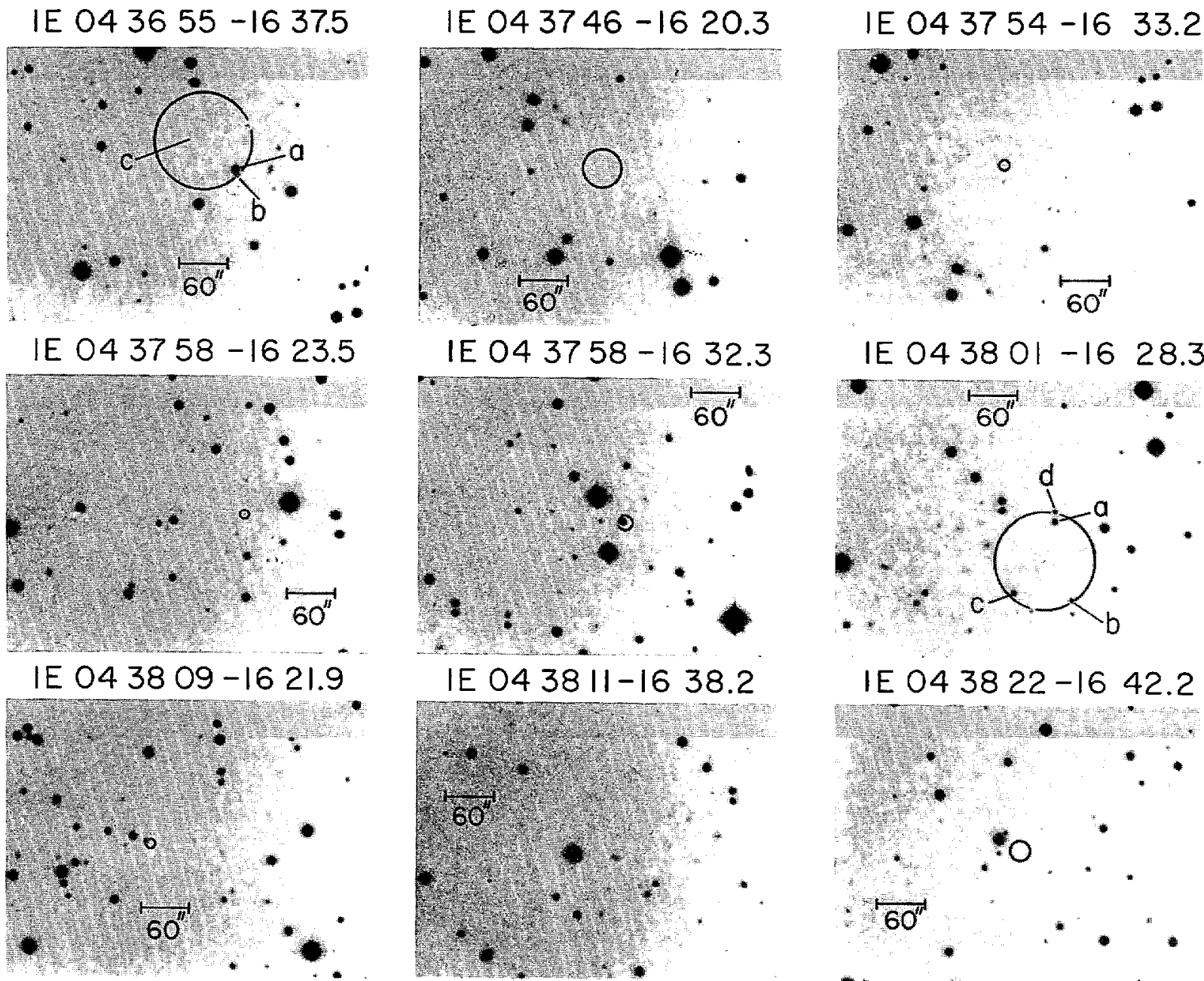

IE $043826-1635.6$
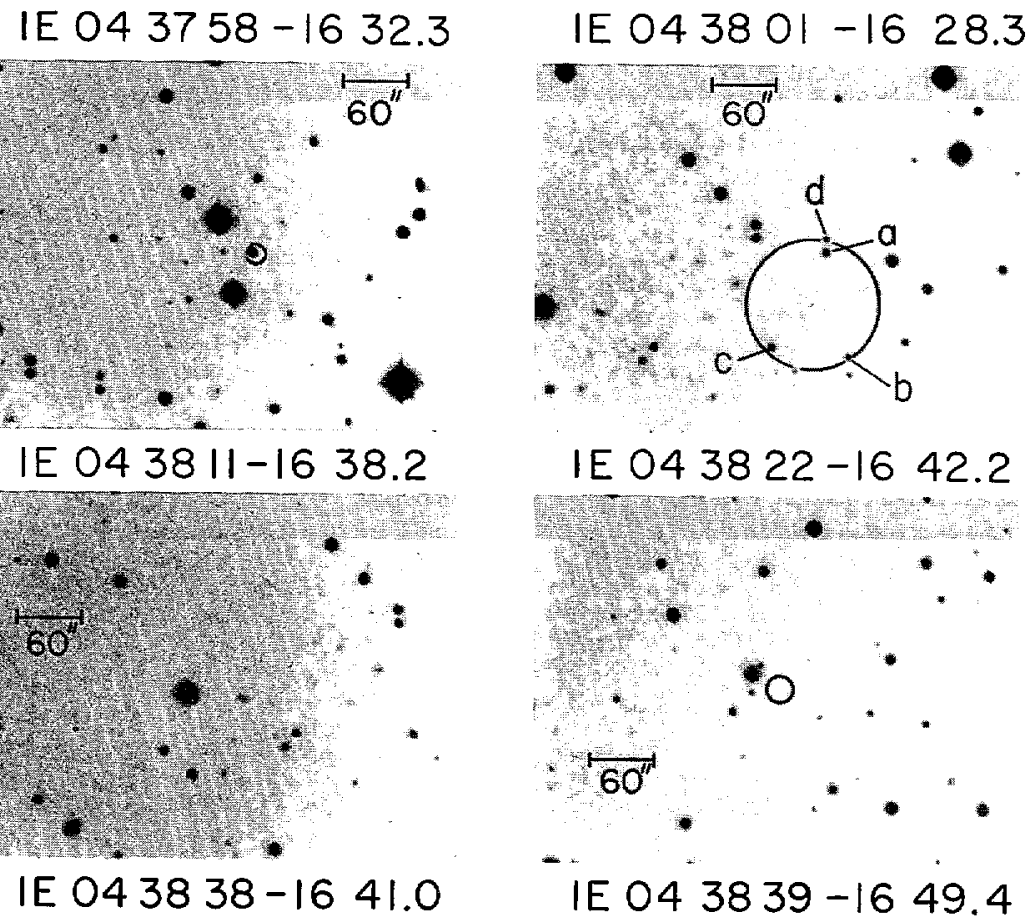

Fig. 3.-Finding charts for sources in the constellation Eridanus, as listed in Table 2

GiacconI et al. (see page L2) 

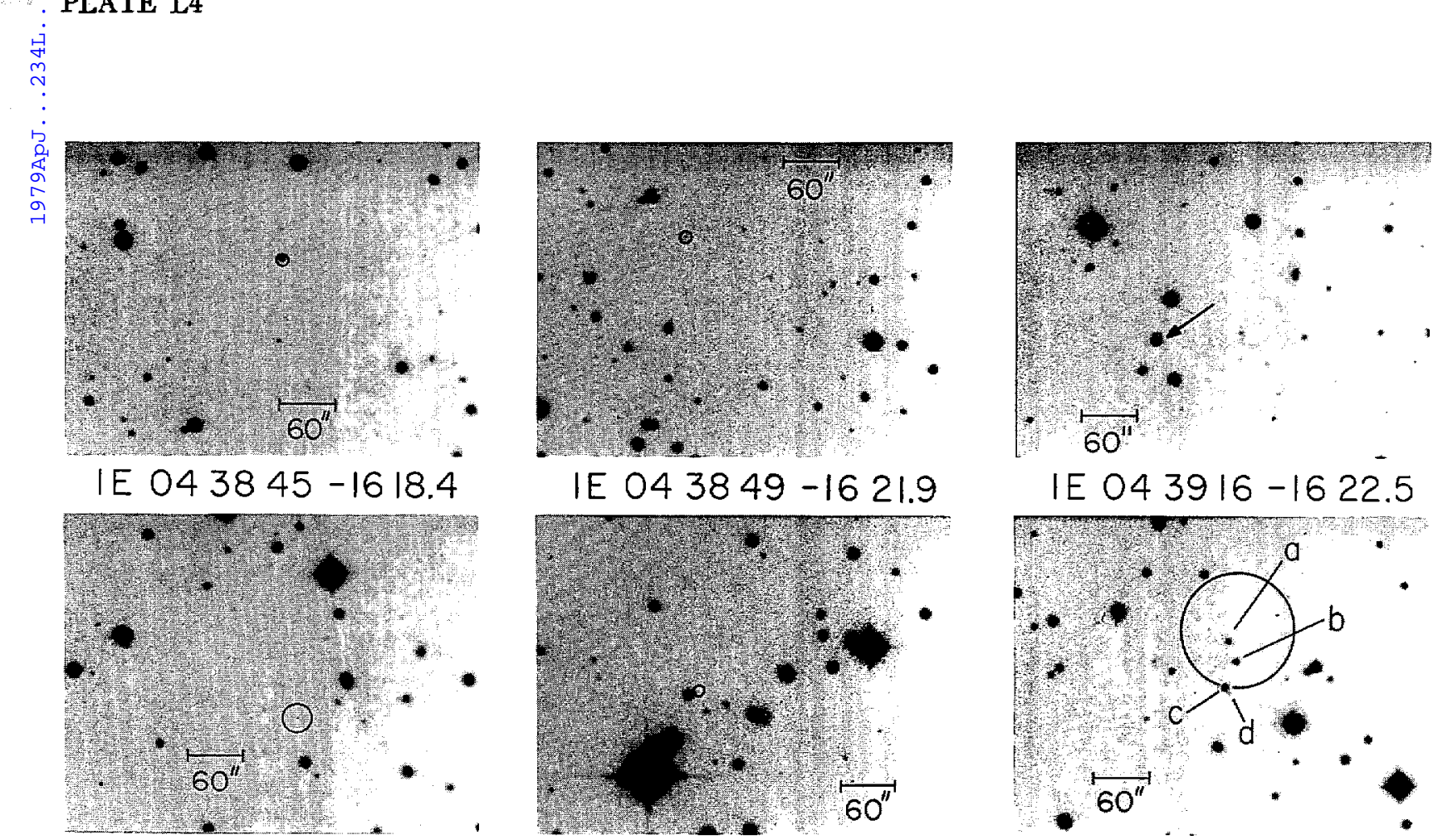

IE $043716-1635.2$

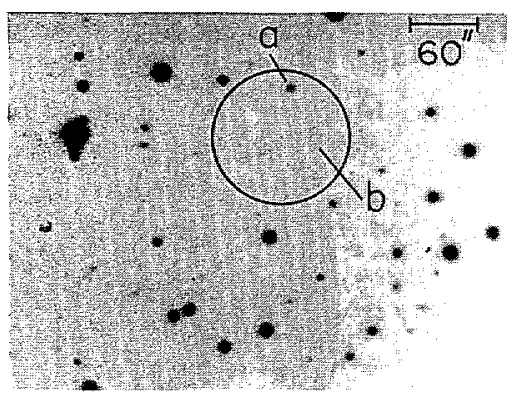

IE $043753-1612.3$

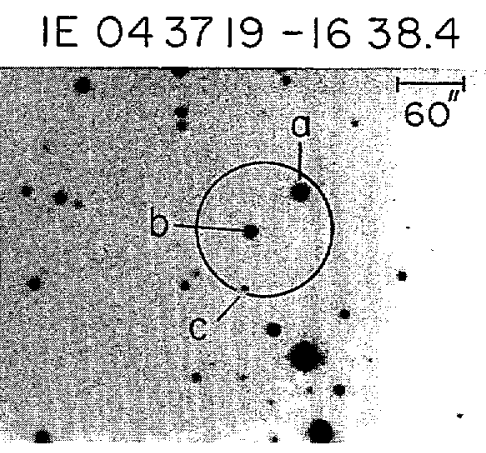

IE $043814-1635.4$
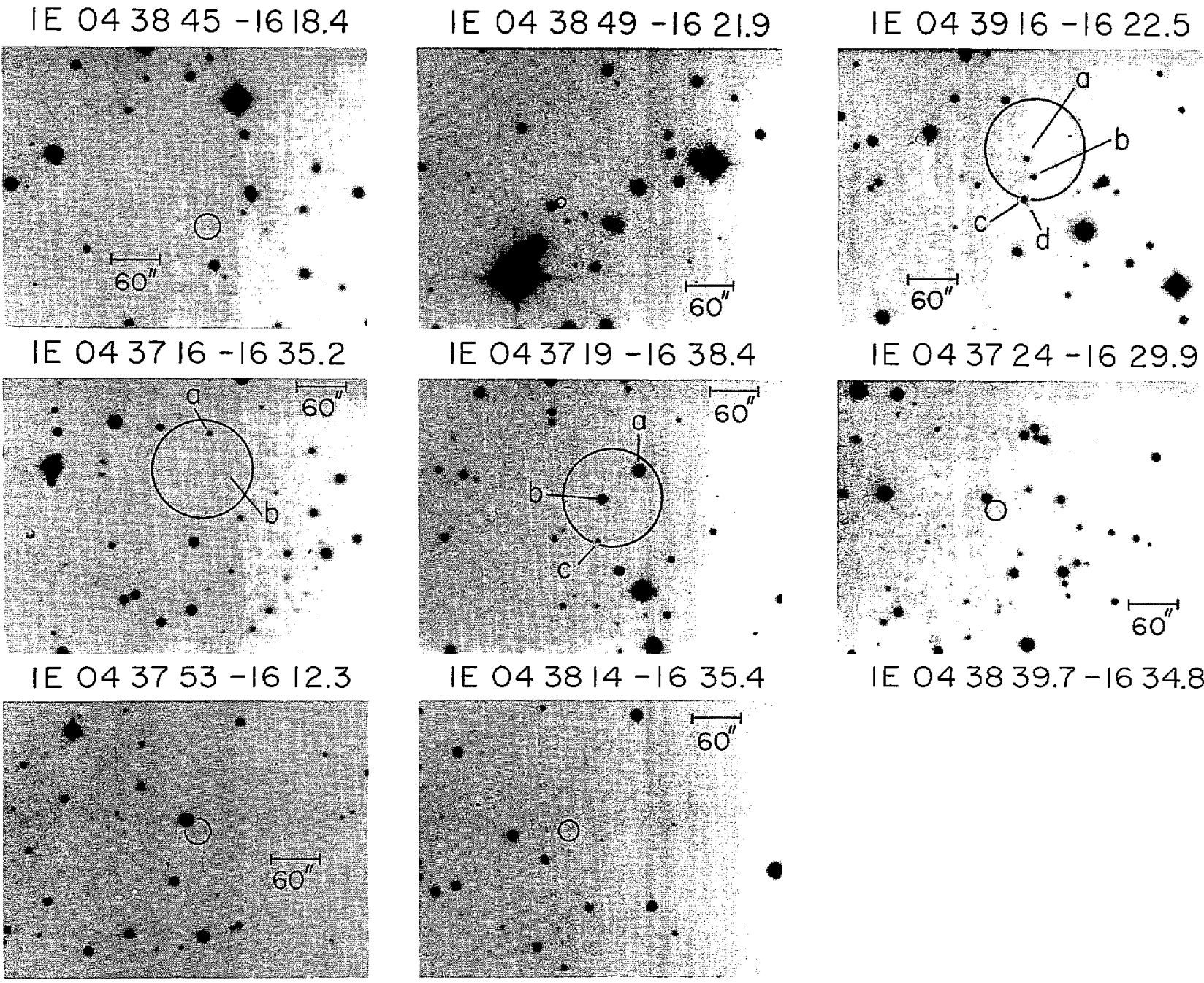

IE $043947-1620.5$

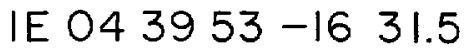

FIG. 4.-Continuation of finding charts for sources in the constellation Eridanus, as listed in Table 2

GiAcconi et al. (see page L2) 